\title{
Impulso formal e impulso sensible, algunas consideraciones sobre la educación estética desde Friedrich Schiller*
}

\section{Formal and Sensuous impulses: some considerations on Friedrich Schiller's aesthetic education}

Jorge Gregorio Posada Ramírez
Profesor Asociado de la Universidad del Quindío. Miembro del grupo de investigación Razones y Acciones de esta misma universidad (clasificación en D por Colciencias). Correo electrónico gposada@uniquindio.edu.co

Cómo citar: Posada, J.G. (2016) Impulso formal e impulso sensible, algunas consideraciones sobre la educación estética desde Friedrich Schiller. Sophia 12 (2): 279-289.

\section{Resumen}

Este texto es un artículo de reflexión. Argumenta que la educación estética es un promisorio camino para promover uno de los más importantes objetivos de la educación: la formación integral. A partir de las Cartas sobre la educación estética del hombre, del filósofo y poeta Friedrich Schiller, se muestra que dos son los rasgos que pueden definir la vocación intelectual del hombre: el impulso formal y el impulso sensible. El primero, recoge la necesidad humana de racionalidad, esto es, la vocación a encontrar y construir abstracciones y regularidades del mundo, de fundirse en lo universal; el segundo, la inclinación de las personas a buscar emociones que aviven su sensibilidad y pasiones, de mantenerse como seres singulares. Descrito el impulso formal y el impulso sensible como las dos cualidades que holísticamente configuran las potencialidades intelectuales humanas, se muestra que la apreciación de lo bello, en términos de Schiller: la Forma viva, en tanto cultiva equilibradamente ambos ímpetus humanos, fomenta la educación integral en las personas.

Palabras clave: Belleza, formación integral, forma viva, impulso formal, impulso sensible, Schiller.

Abstract

This text is a reflective article. It argues that aesthetic education is a promissory path for promoting one of the most important educational objectives: comprehensive training. Starting from the letters On the Aesthetic Education of Man written by the philosopher and poet Friedrich Schiller, it is shown that there are two traits that can define the intellectual vocation of a man: the formal and the sensuous impulse. The first one gathers the human necessity for rationality: the vocation to find and build abstraction and regularity from the world, to merge with the universal. The second one, deals with the preference of people for finding emotions that fuel their sensibility and passion, to remain as singular beings. Having described the formal and sensuous impulses as the two qualities that holistically configure the human intellectual potentialities, we show that the appreciation of beauty, or as Shiller called it the living form - as it evenly nurtures both human impetus- fosters comprehensive training for people.

Keywords: Schiller, formal impulse, sensuous impulse, beauty, comprehensive training, living form.

\footnotetext{
* El autor da los créditos y agradece a la Universidad del Quindío (Colombia) por el apoyo recibido para la realización de este producto académico. Algunas ideas de este artículo han sido motivadas en el marco del Doctorado en Literatura de la Universidad Tecnológica de Pereira (Colombia), en especial, en el Seminario de Investigación Permanente, orientado por el profesor e investigador Eduardo Subirats. Sea esta la oportunidad de dar los agradecimientos; aunque como es de suponerse, los posibles errores en las ideas expuestas son responsabilidad exclusiva del autor.
} 
Al cabo de los años he observado que la belleza, como la felicidad, es frecuente. No pasa un día en que no estemos, un instante, en el paraíso.

Jorge Luis Borges

\section{Cartas sobre la educación estética del hombre: una anatomía estética sobre el intelecto humano}

Cartas sobre la educación estética del hombre (1795) del filósofo, poeta e historiador Friedrich Schiller es uno de esos ensayos que bien puede enarbolarse como un bien universal de la humanidad. Escrito con el propósito de dilucidar los asuntos centrales referentes al arte y la belleza, Schiller concibe un texto que ordena y sintetiza algunas de las ideas más importantes que durante la Ilustración y la segunda mitad del siglo XVIII se cristalizaron para entender el lugar del hombre en el mundo. Teniendo como trasfondo la filosofía de Immanuel Kant, Schiller no solo escribe un magnifico texto estético, del que dice Goethe: "Lo sorbí de un solo trago" (Safranski, 2011:138), sino que decanta algunas imprescindibles ideas para entender las disposiciones morales, intelectuales e instintivas de la vida humana. Menos popular que el ensayo de Kant ¿Qué es la ilustración? (1784) pero igual de contundente y comprometido con la descripción de las facultades intelectuales humanas, Cartas sobre la educación estética del hombre, describe con serenidad, profundidad y un original estilo poético, las recurrentes tensiones que se dan entre las facultades de la razón y la sensibilidad, pero a su vez, la armonía que alcanzan estas facultades una vez que se amalgaman en el arte y en la apreciación de la belleza.

La tesis central de Schiller es que el cultivo del arte permite potenciar el desarrollo integral de las personas, pues, la razón y la sensibilidad, las dos cualidades dominantes del ser humano, logran un despliegue equilibrado una vez que se dirigen a la producción de obras de arte y a la contemplación de la belleza del mundo. El uso mesurado de la razón en su conexión con la sensibilidad permite vislumbrar una vida conforme al ideal elevado de humanidad. Privilegiar una facultad en detrimento de la otra, como suele suceder con los sistemas políticos excesivamente legalistas o los programas académicos desmedidamente cientificistas no solo desvía al hombre de su aspiración a una vida social justa, sino que también deforma y frustra la vida de cada una de las personas. Dice Schiller en sus Cartas:

\begin{abstract}
Pero el hombre puede oponerse a sí mismo de dos maneras: o bien como salvaje, si sus sentimientos dominan sus principios; o bien como bárbaro, si sus principios destruyen a sus sentimientos. El salvaje desprecia la cultura y considera la naturaleza como su señor absoluto; el bárbaro se burla de la naturaleza y la difama, pero es más despreciable que el salvaje, porque sigue siendo en muchos casos el esclavo de su esclavo. (Schiller, 1795: 4).
\end{abstract}

Para Schiller, la facultad de la razón está avocada a establecer principios, reglas generales, que prescriben a partir de razonamientos abstractos, la validez de las experiencias cognitivas y la corrección de las acciones morales. La tendencia innata de la razón a la universalidad conduce a que se le dé la espalda a los hechos particulares y a los contextos específicos en los que ellos devienen. Seguir a rajatabla las leyes de la razón es negar lo que está en juego en las vivencias y sucesos particulares. Es estar dominado por el impulso formal de la razón, esto es, la propensión a seguir reglas y principios universales como únicas formas de cognición y actuación. Schiller llama bárbaros a los que sin consideración de las circunstancias siguen los principios emanados de la razón pura. Los bárbaros son los enajenados por el impulso formal. Los salvajes son aquellos a los que las pasiones primitivas gobiernan sus vidas. Incapaces de contener las emociones y sensaciones provocadas por las excitaciones del mundo, los salvajes no tienen la fortaleza para seguir las reglas construidas desde la razón. Schiller llama impulso sensible a la necesaria avidez humana de ser afectado sensorialmente por la realidad material. La exigencia de vivencias variadas, de emociones no comprimidas y reguladas por el hábito o la razón, es una de las manifestaciones del impulso sensible. Los salvajes son los que están plenamente robados por el impulso sensible.

Si bien Schiller no clasifica a todas las personas como necesariamente bárbaras o salvajes, desde acá puede verse los lados extremos que para él configuran la vida intelectual de las personas: razón y sensibilidad: impulso formal, impulso sensible, y en sus usos 
desmedidos y viciosos: barbarie y salvajismo. La moderación y uso armónico de las dos facultades las postula Schiller en el arte, entendido tanto como capacidad de producir obras bellas, como de apreciar y dejarse afectar por la belleza.

Antes de describir cómo vincula Schiller a la razón y la sensibilidad en el arte y la belleza, y con ello, cómo podría postularse la educación estética, como un impulsor humano de las reglas racionales y de las pasiones moderadas, es pertinente mostrar un caso, construido desde la literatura, de alguien que se deja llevar en un momento de su vida por un enérgico uso de la razón y en otro momento por la pura sensibilidad. Gustavo Aschenbach el personaje central de la novela de Thomas Mann La muerte en Venencia (1983) ejemplifica el dominio de la razón laboriosa y de la sensibilidad avivada, a las que se refiere Schiller como parte fundamental de la naturaleza humana. Sin ser un bárbaro o un salvaje, Aschenbach es un posible arquetipo de estas dos fuerzas que definen la vida integral del intelecto humano: el impulso formal y el impulso sensible.

\section{Gustavo Aschenbach: razón laboriosa y sensibilidad avivada}

En las páginas iniciales de la magistral novela de Thomas Mann, La muerte en Venecia (1983) se representa el que podría ser el ideal del hombre virtuoso al que aspira la educación proyectada por el Estado. El protagonista de la novela, Gustavo Aschenbach, es un hombre que ha merecido el reconocimiento de las gentes por su honesto y laborioso trabajo como escritor. Tiene la capacidad de controlar y dirigir adecuadamente los impulsivos deseos humanos causantes, en los de su generación, del egoísmo, frivolidad y brutalidad que ha definido a los de su época. En sus obras, producto no del natural y espontaneo talento, sino de la trabajada disciplina vuelta hábito, se resaltan personajes que encarnan valores morales necesarios para la correcta formación de las nuevas generaciones. Sus libros, sin ser moralistas, son incluidos en las escuelas como parte de los textos formativos que los estudiantes deben de leer. La certeza de que el trabajo constante y el dominio de las pasiones es la vía para alcanzar una vida justificada, la resume Aschenbach cuando en uno de sus libros escribe:
Casi todas las cosas grandes que existen son grandes porque se han creado contra algo, a pesar de algo: a pesar de dolores, y tribulaciones, de pobreza y abandono; a pesar de la debilidad corporal, del vicio y de la pasión. (Mann, 1983: 20).

Como fórmula, no solo para su trabajo como escritor sino también para enfrentar la vida misma, en las páginas iniciales de La muerte en Venencia, Thomas Mann crea un personaje que bien puede servir para modelar la capacidad de la educación en la formación de ciudadanos: un profesional honesto que conoce con profundidad su oficio, ejecutándolo por el bien de los otros. Pero, Aschenbach, una vez que decide salir de vacaciones para airear su rutina laboral, se enfrenta con la visión de la belleza, en la que es para él su forma más pura y elevada. Aschenbach ve en un hotel de Venencia a un joven polaco de una hermosura tan sobrecogedora que no duda en asumirlo como el ideal platónico de lo bello. Tadrio un joven de catorce años le hace emprender a Aschenbach un nuevo camino en su vida, el de la sensibilidad intelectual que es estimulada ante la percepción de lo bello. No hay un asomo de lascivia en la relación meramente contemplativa de Aschenbach hacia Tadrio. Su belleza le parece tan superior que no es capaz de vencer la timidez para cruzar unas cuantas palabras con él. La juzga como el bien espiritual más elevado al que los humanos pueden aspirar a través de la percepción sensorial. En referencia a Sócrates afirma Aschenbach pensando en la hermosura de Tadrio:

Porque la belleza, Fedón, nótalo bien, solo la
belleza es al mismo tiempo divina y perceptible.
Por eso es el camino de lo sensible, el camino
que lleva al artista hacia el espíritu. Pero ¿crees
tú, amado mío, que podrá alcanzar alguna vez
sabiduría y verdadera dignidad humana aquel
para quien el camino que lleva al espíritu pasa
por los sentidos? ¿O crees más bien (abandono
la decisión a tu criterio) que éste es un camino
peligroso, un camino de pecado y perdición,
que necesariamente lleva al extravío? (Mann,
1983:136).

Si bien Aschenbach renuncia a la controlada y calmada vida que llevaba para poder continuar sintiendo el gocé estético e intelectual que le genera la visión de Tadrio, su figura representa los dos componentes esenciales que condicionan la vida intelectual de las personas: el impulso formal y el impulso sensible, en 
términos de Schiller. La determinación a conducirse siguiendo preceptos racionales, y a su vez, la inclinación a consumir experiencias sensibles es el motivo central de la novela. Razón y sensibilidad son las dos facultades que configuran la vida mental de las personas, y las que se presentan en el personaje principal de La muerte en Venencia de Thomas Mann. Inicialmente Aschenbach encarna el arrobo, aunque no vicioso, del impulso formal. Las apetencias que corrientemente afectan a los hombres y les hacen ir más allá de sus propios deseos de control, él las reducía a: "Justas proporciones por la razón y por el dominio de sí mismo, adquirido a fuerza de ejercicios." (Mann, 1983: 11). Pero una vez que ve al hermoso Tadrio y que reconoce en él una poderosa fuente de emociones sensoriales, Aschenbach es secuestrado de su temperancia racional y se pierde en el incontenible torrente del impulso sensible. Thomas Mann describe las percepciones de Aschenbach cuando narra:

La visión de aquella figura viviente, tan delicada y tan varonil al mismo tiempo, con sus rizos húmedos y hermosos como los de un dios mancebo que, saliendo de lo profundo del cielo y del mar, escapada al poder de la corriente, le producía evocaciones místicas, era como una estrofa de un poema primitivo que hablara de los tiempo originarios, del comienzo de la forma y del nacimiento de los dioses. (Mann, 1983: 63-64).

Hasta ahora tenemos que desde Schiller dos son las facultades fundamentales para comprender la vida intelectual del hombre: razón y sensibilidad, y con ellas, dos fuerzas que impelen a sus respectivos usos: el impulso formal y el impulso sensible. La convergencia armónica de estas es la aspiración a una educación humana integral. Pues cuando se privilegia una de las facultades, uno de estos impulsos, en detrimento del otro, se castra una condición natural del humano, arrobándolo a la barbarie, si se potencia solo la razón, o a salvajismo si solo se atiende los impulsos sensibles. Como caso representativo de un hombre que encarna la disposición intelectual de estas dos facultades, se describió a Gustavo Aschenbach, personaje principal de la novela La muerte en Venecia de Thomas Mann. Al inicio de la novela Aschenbach se presenta como un hombre condicionado por el impulso formal, y al final, y una vez que es estimulado por la visión de la belleza, es enajenado por el impulso sensible.
En lo que sigue, se ampliará la distinción de las facultades intelectuales que propone Schiller. A partir de su fuente filosófica directa: Immanuel Kant, se analizará la actividad de la razón y de la sensibilidad. Se mostrará cómo una reverencia excesiva al desarrollo de la razón, parece marcar el interés de formación por parte de las instituciones educativas. Finalmente, y retomando a Schiller, se argumentará cómo la formación estética: la capacidad para apreciar la belleza, es una promisoria manera de formar un carácter armónico e integral, pues en la capacidad para dejarse afectar por lo belleza genuina se da un adecuado balance entre el impulso formal y el impulso sensible.

\section{Impulso formal e impulso sensible}

El deseo de comprender los mecanismos que constituyen y mueven la vida intelectual humana, encontró en la filosofía moderna su momento de mayor auge y desarrollo. Las dos grandes corrientes filosóficas modernas: racionalismo y empirismo, ofrecen atractivas e influyentes respuestas a las preguntas cómo está constituido el intelecto humano y cuál es su manera de aprehender y tener conocimiento del mundo. El filósofo francés Rene Descartes, el más popular de los racionalistas, afirma que es en los procesos racionales y formales como la facultad del intelecto se despliega, obteniendo el conocimiento de las cosas (2010). El filósofo escocés David Hume, el más congruente de los empiristas, muestra que es a través de la información de los sentidos como se determina el impulso y los alcances de toda la vida intelectual humana (2008). Pero fue Immanuel Kant el que ofreció la respuesta más completa e influyente sobre cuáles son los límites y las facultades del intelecto humano. Posterior a Descartes y Hume, Kant sintetizó en un sistema coherente las ideas menos controvertibles del racionalismo y del empirismo moderno. Su explicación sobre los límites y capacidades del intelecto humano es expuesta en la obra Crítica de la razón pura sin lugar a dudas una de las más destacadas obras de la filosofía occidental. En ella, y sin quedarse en la mera integración del racionalismo y el empirismo, Kant logra revelar los elementos y principios esenciales del intelecto humano. 
Schiller afirma que a pesar del tecnicismo de la filosofía kantiana, la humanidad siempre ha estado de acuerdo con las ideas de Kant, pues: "Si se les libera de su forma técnica, aparecen como sentencias antiquísimas de la razón común y como hechos de aquel instinto moral que la sabia naturaleza da al hombre como tutor" (Schiller, 1795: 1). Desde Kant y el influjo que el racionalismo y el empirismo da a su filosofía puede comprenderse con mayor claridad los llamados por Schiller impulso formal e impulso sensible del intelecto. Como es de presumirse, desde el racionalismo se puede considerar el impulso formal de la razón y desde el empirismo el impulso sensible. Para Kant, entendimiento y sensibilidad son las dos facultades del intelecto que deciden los límites de todo conocimiento y experiencia posible. En el entendimiento: se concentra la capacidad de la razón para ordenar en abstracciones y leyes el mundo, en la sensibilidad: la disposición del intelecto para dejarse afectar por los objetos del mundo.

En La crítica de la razón pura Kant afirma del entendimiento:

$\mathrm{Si}$ en alguna parte existen principios, ello se debe únicamente al entendimiento puro. Este no solo es la facultad de las reglas relativas a lo que sucede, sino que es la misma fuente de los principios en virtud de los cuales todo cuando se nos puede presentar sólo como objeto se halla necesariamente sometido a reglas (Kant, 2006: 197).

Para Kant la experiencia humana pasa por ordenar conceptualmente la vorágine de sensaciones que asaltan diariamente a los sentidos. La profusión de sensaciones que recibe el sistema perceptual es tan caótica que solo abstrayendo y ordenando en formas comunes este confuso influjo, es posible reconocer o pensar algo. El entendimiento es el encargado de imponer conceptos y esquemas a ese raudal de información sensorial para poder delimitar una experiencia pensable. Así, el entendimiento es la facultad humana que permite el conocimiento conceptual y la comprensión de las sensaciones, toda vez que las ordena en reglas y esquemas. Esta facultad estructura a los datos sensoriales en las formas que necesariamente posibilitan el pensamiento.

De otra parte, la sensibilidad es para Kant la facultad que permite que los objetos afecten o estimulen la psiquis de los humanos. Las personas pueden sentir el flujo del mundo externo, pueden ser impresionadas por las cosas que los rodean, gracias a la facultad de la sensibilidad. Kant la entiende como una facultad receptiva, en tanto su principal función es recibir y presentar, en forma de sensaciones, los objetos y hechos. La manera inicial como el mundo se manifiesta a las personas es a través de las percepciones sensoriales, todas ellas, dominio de la facultad de la sensibilidad. En Kant, el modo como la mente humana es afectada por la realidad pasa inicialmente por la sensibilidad, la cual define como: "La capacidad (receptiva) de recibir representaciones, al ser afectados por los objetos se llama sensibilidad. Los objetos nos vienen, pues, dados mediante la sensibilidad y ella es la única que nos suministra intuiciones." (2006: 65)

Para Kant espacio y tiempo son las formas básicas de la sensibilidad. Todo objeto que impresione la sensibilidad necesariamente ha de estar sometido a las determinaciones de espacio y tiempo. Así, la facultad de la sensibilidad impulsa la actividad intelectual humana una vez que recoge el material de las sensaciones dado en espacio y tiempo. De esta forma, es la facultad que otorga el material del que se vale el entendimiento para ejercer su actividad de síntesis y orden. Ella recibe y a su vez da el contenido que es conceptualizado y estructurado en el entendimiento. En relación con este último, la sensibilidad es una facultad pasiva, pues recibe y trasfiriere sensaciones al entendimiento, mientras que este es activo, pues configura en reglas y formas los datos suministrados por la sensibilidad. En esencia, la más alta labor de la especie humana: pensar, consiste ordenar en conceptos (entendimiento) lo que nuestros sentidos reciben de los objetos (sensibilidad). De ahí la sentencia de Kant: "Los pensamientos sin contenido son vacíos, las intuiciones sin conceptos son ciegas." (2006: 93).

Algunas de las ideas estructurales de las Cartas sobre la educación estética del hombre revelan una gran influencia de la filosofía de Kant. Sin hacer copia de las tesis kantianas, Schiller logra mostrar cómo el sistema de Kant le da profundidad y universalidad, no solo a una teoría estética, sino a una propuesta de formación humana. Al explicar Kant los límites y capacidades del intelecto humano, sugiere cuáles 
son los campos mentales que deben ser abonados y controlados para potencializar la más noble capacidad de las personas: el pensamiento. Schiller se refiere a Kant como el más grande filósofo de su época (2011), y lo es también para la teoría de Schiller, pues el impulso formal y el impulso sensible son la interpretación viva y dinámica del entendimiento y la sensibilidad de Kant.

La necesaria capacidad humana de recoger y unificar los rasgos comunes de los objetos para obtener su comprensión, es una inclinación que va más allá de la mera existencia mental de cada uno de los individuos. La disposición del entendimiento a la abstracción es llevada a la vida pública y política a través de los ideales que el Estado traza para la vida social de las personas. Así como en el concepto "silla" el entendimiento unifica una variedad de objetos del mundo, sin tomar de estos sus colores, texturas, formas y materia, el Estado unifica en unas ideas rectoras los modos acción de la gente. Para Schiller esto es posible porque existe en el hombre una disposición a la universalidad, esto es, a sumirse en ideas que diluyen la singularidad de su vida, que lo arrebatan de sus determinaciones espaciales y temporales. Para Schiller este es el hombre ideal. Dice:

A este hombre puro, que se da a conocer con mayor o menor claridad en todo sujeto, lo representa el Estado, que es la forma objetiva y, por así decir, canónica, en la que trata de unirse la multiplicidad de los sujetos. (Schiller, 1795: 4).

Así, en tanto el Estado como los individuos son impelidos por la necesidad de abstracción, estos se conducen hacia el seguimiento de ideas universales, reglas generales que homogenizan gran parte de sus vidas. El impulso de abstracción, que como se mostró en términos de Kant, es la disposición del entendimiento a organizar en conceptos y esquemas la diversidad, es descrito por el Schiller no solo como parte del intelecto humano, sino como el método del Estado para organizar a los individuos. Puede afirmarse que las instituciones políticas son posibles dado que cada individuo porta en sí un hombre ideal, y gracias al impulso de abstracción que permite que las personas construyan y sigan reglas universales desde las que se satisface al hombre ideal. La adherencia de las personas a un partido político, a una religión, a una ideología son casos representativos de la existencia del llamado por Schiller hombre ideal y su consecución a través del impulso formal.

El intelecto exige también variedad. Para Schiller, junto al hombre ideal reposa el que puede denominarse el hombre temporal. A diferencia de aquel, este recoge la necesidad humana de singularidad. Las exigencias que impone la facultad de la sensación de tener emociones y pasiones, de ser estimulada por los objetos, avoca al hombre a sumergirse en los lugares y tiempos específicos en que ocurren los sucesos. Así, una condición necesaria de la sensibilidad es habitar y seguir los llamados de un espacio y tiempo específico, desatendiendo el deseo de universalidad. De hecho, y como se mostró desde Kant, la abstracción propia del entendimiento, solo es posible en tanto la sensibilidad recoja la variedad de las impresiones causadas por los objetos. Estos, en tanto determinados en espacio y tiempo, subyugan al hombre a cohabitar en el espacio y tiempo específico en el que se encuentran. El hombre temporal es para Schiller el modo de ser de las personas que es condicionado por el impulso sensible, es el deseo de sentir y vivir en el espacio y tiempo presente, de ser un sujeto singular, no universalizable.

Desde el punto de vista integral, para Schiller el hombre es un ser ideal y un ser temporal, un individuo que busca la universalidad, y a su vez, la singularidad. A través del impulso formal construye reglas e ideas que satisfacen su vocación a la abstracción; desde el impulso sensible, se deja estimular por los sucesos de su espacio y tiempo específico, atendiendo su deseo de singularidad. Dice Schiller: "El impulso sensible exige que haya variación, que el tiempo tenga un contenido; el impulso formal pretende la supresión del tiempo, que no existe ninguna variación." (Schiller, 1795:21). La vocación intelectual del hombre requiere satisfacer ambos impulsos. Más que sugerir dos modos de ser distintos y excluyentes, muestran su fuerza de complementariedad. Forma y contenido son las categorías que están en la base fundamental de la propuesta de Schiller. Solo habrá forma en tanto la materia esté configurada, y solo habrá contenido en tanto se pertenezca a una forma. 


\section{El impulso formal sobre el impulso sensible: un desequilibrio latente}

Ninguno de estos dos tipos de ímpetus, que constituyen la vida mental de las personas, debe superponerse al otro. Pero Schiller llama la atención a la sobrevaloración del impulso formal en la educación civil e intelectual del hombre. La necesidad del Estado de universalizar la existencia de las personas, para intentar hacer de la vida colectiva un todo integral, ha hecho que se privilegie las formas de vida universales, en detrimento de las individualidades. El hombre ideal en su deseo de abstracción y generalidad fácilmente sigue las ideas construidas desde el Estado o desde las instituciones que tienen el poder de masificar ideas. Si las ideas del Estado o de las instituciones son promisorias para la vida integral de las personas, el deseo de abstracción del hombre es correctamente satisfecho. Pero si estas ideas, como lo muestra Schiller, niegan el despliegue armónico de la vida sensible de las personas, el resultado es la frustración y el caos existencial de los individuos y de la sociedad. En esencia, un carácter trastornado es el resultado de un desequilibrio en uno de los impulsos intelectuales humanos. Para Schiller, tanto en los individuos como en la sociedad puede verse el carácter perturbado. Por lo que es apremiante comprender en dónde radica el desequilibro de los impulsos intelectuales.

"El hombre se refleja en sus hechos; y iqué espectáculo nos ofrece el drama de nuestro tiempo" (Schiller, 1795:5). Con esta frase Schiller condesa el estado actual, no solo de su época, sino de todo momento de la historia en el que uno de los dos impulsos intelectuales deforma la vida integral del hombre. Indiferencia, apatía, masificación de las ideas, insensibilidad hacia el dolor de los otros, frivolidad, sobrevaloración a cualquier tipo de ideas: religiosas, políticas, raciales más que a las personas, son el signo del caótico carácter de la época. El interés del Estado y las instituciones políticas y económicas de dominar a las personas ha exacerbado el impulso formal de la época. Seguir preceptos universales, sin reflexionar sobre su genuina conveniencia es el signo de estos días. La sensibilidad de los individuos se rinde ante ideas ajenas a sus verdaderos quereres. La gran denuncia de Schiller a su época, que en parte puede replicarse a nuestros días, es el excesivo control racional, por parte de agentes externos, a la vida de las personas. Y este control no necesariamente es punitivo, obedece a la necesidad irreflexiva de seguir ideas, abstracciones que niegan el ser individual, el hombre temporal que hay en cada uno de los sujetos. Escribe Schiller:
Si la colectividad hace de la función pública la medida del hombre, si aprecia a uno de sus ciudadanos sólo por su memoria, al otro por su inteligencia tabular, y a un tercero únicamente por su habilidad mecánica; si en un caso, sin tener en cuenta el carácter, insiste sólo en los conocimientos, y en otro, por el contrario, acepta incluso su inteligencia menos lúcida, mientras se trata de un espíritu de orden y se conduzca según la ley; si pretende al mismo tiempo que esas habilidades individuales se desarrollen tan intensamente, como mínima es la extensión que se le permite al propio individuo, ¿nos extrañara entonces que se desatienda las restantes facultades espirituales, para dedicar todas las atenciones a la única que proporciona consideración social y que resulta ventajosa? (Schiller, 1795: 7).

La especialización de las vidas, de cuenta del interés del Estado de ordenar en una totalidad a los individuos, es para Schiller una de las formas de frustración y desequilibrio de las personas. La estructura social vista como un gran mecanismo requiere que cada sujeto cumpla tareas específicas. Al asumirse las vidas solo en función al mantenimiento de la totalidad colectiva, se niega al individuo y se distorsiona su impulso sensible, aquel que le permite reconocerse como perteneciente a un lugar y tiempo específico y sentirse como un ser dotado de singularidad. El hombre temporal, y con él, el impulso sensible es cercenado al verse el hombre arrastrado a seguir ideas y valores impuestos por su época. Más que un signo de esta época, es un hecho incontrovertible la dependencia de la sociedad a la especialización de los individuos. Al tener que sumergirse el hombre en un solo compartimento dentro de la totalidad social, se fragmenta su ser y su capacidad de reconocer y elegir sus propios deseos y pasiones. Hasta el uso del ocio es manipulado por la fuerza abstractiva de las instituciones que deciden las ideas rectoras de los sujetos. Para Schiller, solo un Estado que logre leer las tendencias singulares de sus ciudadanos, y desde ellas, construir los preceptos generales que los guíe como pueblo, puede encausar armónicamente el impulso sensible y el impulso formal. 
No cree Schiller que sea una idea utópica la armonía entre el hombre ideal y el hombre temporal, pues al ser habitantes de una misma época, los modos de sensibilidad de los individuos no necesariamente son inconmensurables. Además, se pueden educar adecuadamente el carácter de las personas, en especial, su impulso formal. Sobre la base de que existen formas universales, estructuras abstractas no decididas por el arbitrio de los gobernantes o instituciones de turno, se puede trazar un camino que predisponga a los sujetos a abstracciones que no rivalizan con la sensibilidad. Para Schiller a pesar de lo enturbiadas que pueden ser las épocas de la historia humana, dos ideas rectoras siempre se imponen: la belleza y la verdad. Estas no dependen de los sujetos, en el sentido de que no son creadas a merced de los intereses o caprichos de las personas. Son ideas universales que muestran en su constitución el elemento formal y su contenido, impulso formal e impulso sensible. Para Schiller la verdad y la belleza son parte de los bienes universales, de las nociones ahistóricas que permiten a la humanidad estar unidad en un solo linaje. La fuerza titánica de la verdad y la belleza no se amengua ante la frivolidad y apatía de los tiempos, y es en ellas, en especial en la belleza que es forma y contenido, desde donde se puede educar el carácter de las personas. Schiller sentencia:

Durante siglos, tanto los filósofos como los artistas han tratado de hacer llegar la verdad y la belleza a las clases más bajas de la humanidad: ellos fracasaron en el intento, pero la verdad y la belleza se abrieron camino victoriosamente gracias a su propia fuerza vital indestructible. (Schiller, 1795: 12).

La universalidad de las formas y contenidos de la belleza es sustentada por Schiller al afirmar que no es propiedad de unos cuantos la sensibilidad ante las formas bellas, pues, al estar constituida por el impulso formal y el impulso sensible, y al ser ambos impulsos disposiciones connaturales de las personas, la apreciación sensible hacia las formas bellas es universal. Puede argumentarse, entonces, que desde Schiller en la belleza se da un camino promisorio para la educación equilibrada entre el hombre ideal y el hombre temporal. Esto porque la belleza es la incontrovertible armonía entre contenido y forma, sensibilidad y entendimiento, singularidad y universalidad. Schiller llama forma viva a un tercer impulso presente en la vida humana. Este se configura cuando hay un natural y espontaneo acuerdo entre el impulso formal y el impulso sensible. Desde la forma viva que objetivamente se manifiesta en las obras bellas o en las cualidades bellas de las cosas propone Schiller la formación equilibra del carácter humano.

La tesis de Schiller es simple: Una educación integral debe cultivar el carácter de manera equilibrada, como el carácter depende del impulso formal y el impulso sensible, la capacidad de reconocer la armonía entre estos dos impulsos es una provechosa vía a la educación integral. Ahora bien, en tanto en las obras bellas se da un equilibrio genuino entre el impulso formal y el impulso sensible, la educación a la contemplación de las obras bellas es un ventajoso camino para la consecución de individuos integrales.

\section{La belleza como forma viva}

Schiller detalla la belleza como forma viva. Si bien, esta definición parece una solución ad hoc para hacer consistente su idea de que a través de la belleza se logra un equilibrio entre el impulso formal y el impulso sensible, Schiller no parte de nociones arbitrarias. Influido por las aceptas tesis de Kant sobre el entendimiento y la sensibilidad, y como se mostró, validado por las experiencias que un hombre puede vivir cuando orienta su existencia a través de reglas racionales o de los impulsos sensibles, como es el caso de Aschenbach, Schiller ve en la belleza un equilibrado acuerdo entre la forma y el contenido, entre el entendimiento y la sensibilidad.

En la expresión forma viva, la noción de forma alude a las relaciones conceptuales, regladas por el entendimiento, y que están implícitas en las cosas a las que les viene el predicado de bellas. Una obra musical, un poema, un verso, una obra literaria, la consecución magistral de una acción como modelar un espacio o resolver un problema, casos de este tipo a los que les viene adecuadamente la palabra bello, revelan necesariamente una forma, el impulso formal. Al decir que es una obra musical bella, un poema bello, una novela bella, un diseño bello o la bella resolución a un problema se acepta implícita o explícitamente que los objetos o acciones evaluadas contienen una estructura, una forma en la que deviene su contenido. La forma y su relación armónica con el contenido es condición necesaria de la belleza. No hay belleza sin forma. 
La belleza causa una agradable impresión sensible. Conduce a un espontanea vivacidad sensorial en el que la percibe. En la expresión forma viva el término viva recoge la necesaria participación de la facultad de la sensibilidad en lo bello. Ya sea como el contenido de la belleza o como el efecto que produce, hay belleza en tanto la sensibilidad participe en el objeto o la acción evaluada como bella. Para Schiller el ser material o la presencia sensible inmediata es lo vivo, y en la belleza se da como el contenido adecuadamente organizado en su forma.

Dos aclaraciones con respecto a la noción de vivo advierten Schiller. La primera, la belleza no se da necesariamente en lo vivo. Una escultura de mármol puede ser bella a pesar de que su materia no lo sea. En la expresión forma viva recoge Schiller la idea de que la materia, independiente de si es viva o no viva, una vez que sigue armónicamente una estructura se hace materia viva. En tanto produzca en el entendimiento como en la sensibilidad una vivacidad luminosa será forma viva, y esto ocurre cuando el contenido y la forma encuentran un cordial enlace. Una escultura de mármol puede ser forma viva y una obra de teatro, inclusive una vida humana, a pesar de que su materia sea viva, no logrará ser forma viva si no hay una correspondencia entre las formas labradas desde el entendimiento y su materia sensible. La segunda aclaración que conviene a las tesis de Schiller es que el hecho de que alguien no pueda conmoverse ante la belleza no significa que ciertos objetos o acciones no sean bellos. La apatía sensorial o el analfabetismo de algunos individuos al reconocimiento de formas bellas, es justamente el resultado del desequilibrio de los dos impulsos intelectuales. Un hombre alienado por el mero deseo de sentir emociones crudas, otro por el excesivo formalismo de la razón, puede tener problemas en apreciar lo bello. La formación estética busca justamente esto, moderar la tendencia excesiva al dominio de uno de los dos impulsos.

La forma viva como definición de lo bello, acentúa la creencia universal y fundamental de que en el mundo existen cosas, hechos, acciones, sucesos y seres bellos. La correcta apreciación de lo bello pasa por un correcto balance entre la forma y el contenido de aquello de lo que se predica que es bello. El equilibrio es necesario pues el intelecto, ya sea del que contempla lo bello o el que lo produce, está restringido a experimentarlo solo cuando los dos rasgos que lo configuran, el impulso formal y el impulso sensible, se amalgaman proporcionadamente. Una mente robada solo por la sensación no aprecia lo bello, igual sucede cuando es secuestrada por la sola forma.

Aceptada la belleza como un hecho real de la humanidad, y su constitución como forma viva, hace que su apreciación se presente como una manera de generar hábitos de armonía entre el impulso formal y el impulso sensible. Un carácter adiestrado para reconocer lo bello podrá estar mejor dispuesto para decidir qué formas del entendimiento le convienen a cierta materia, y cuáles no. De igual manera, qué estímulos sensoriales son ventajosos y cuales subyugantes. Ya sea en el campo político, económico, religioso, escolar la capacidad de armonizar adecuadamente forma y contenido es un posible garante de integralidad de los sujetos consigo mismo y con los otros. Además, ya sea consciente o inconscientemente, la apreciación de la belleza empuja a la consideración de que la vida humana está tejida por sensaciones y formas, por momentos singulares y aspiraciones a lo universal, pues lo temporal y atemporal se trenzan en la belleza. Dice Schiller: "La belleza guía al hombre sensible hacia la forma y hacia el pensamiento; la belleza hace regresar al hombre espiritual a la materia, al mundo sensible." (Schiller, 1795: 27).

\section{Conclusiones}

El loable interés del Estado y de los individuos a la formación integral obliga a reconocer qué es aquello de lo que está hecho el intelecto humano. Apelar a nociones excesivamente minuciosas puede ofuscar la tentativa de un promisorio camino para su consecución. Por esto, nociones que desde su generalidad permiten la consideración de estrategias viables, pueden ser bien recibidas, a pesar de los detalles que dejan por explorar. Desde la obra de Friedrich Schiller Cartas sobre la educación estética del hombre (1795) pudo verse dos grandes rasgos que definen la vida intelectual de las personas: impulso formal e impulso sensible. Se mostró que si bien, estas nociones contienen parte de la doctrina humanística y estética de Schiller, tienen sus antecedentes en la filosofía de Kant, la que lejos de ser un sistema filosófico dogmático, revela buena 
parte de las verdades epistemológicas aprobadas por la humanidad. Por esto, puede considerarse que la obra de Schiller citada en este texto es un valiosísimo documento para entender la vida intelectual de las personas, teniendo como perspectiva su florecimiento integral.

Las ideas de Schiller no se confinan en la mera consideración reflexiva y filosófica. Teniendo como modelo un personaje ficticio, pero a su vez representativo de la humanidad, como son los personajes literarios, se mostró que es posible llevar al mundo de los hechos las reflexiones filosóficas de Schiller. Aschenbach, el personaje principal de la novela de Thomas Mann La muerte en Venecia (1983) fácilmente puede iluminarse interpretativamente desde la noción de Schiller de impulso formal e impulso sensible. Él, como todos los hombres confina su vida a estas dos fuerzas intelectuales; del dominio de una sobre la otra, depende su manera de lidiar con el mundo y con su vida interior. Se mostró entonces que la propuesta de Schiller es válida no solo por la coherencia de sus conceptos, sino también por las vivencias mismas que las personas exhiben.

Apelando a la filosofía de Immanuel Kant se argumentó que el impulso formal de la teoría de Schiller está influenciado por la noción kantiana del entendimiento. $\mathrm{Si}$ el entendimiento en Kant es la facultad activa que ordena y estructura las sensaciones en esquemas, para posibilitar de esta forma el pensamiento, y el impulso formal en Schiller es la disposición humana a reglar a través de nociones abstractas la variedad de percepciones humanas, no es forzada ver la deuda de Schiller a las tesis de Kant. Algo similar ocurre con la teoría kantiana de la sensibilidad. Entendida como la facultad receptiva que permite que el hombre sea afectado por los objetos, la tesis de Schiller del impulso sensible adeuda a Kant. Esta disposición intelectual permite las posibles estimulaciones sensoriales de las personas. Para Schiller el impulso sensible configura al hombre como un ser pasivo en tanto es afectado por los objetos, y un ser temporal, en cuanto solo es posible la activación de este impulso mientras las personas evocan sus determinaciones dadas en el espacio y el tiempo.

Desde la frase de Schiller: "El hombre se refleja en sus hechos; y ¡qué espectáculo nos ofrece el drama de nuestro tiempo" (Schiller, 1795: 5). se asumió que el signo de la época es un desequilibrio entre los dos impulsos que constituyen la vida intelectual. El impulso formal se impone caóticamente sobre el impulso sensible. El excesivo deseo humano de perseguir ideales ajenos al tiempo y el espacio real en que deviene los sujetos, parece probar esto. Apatía, indiferencia, persecución de objetivos genéricos, que obedecen más a la masificación por parte del Estado y los grupos de poder, que al querer propio de los agentes, es una verdad palmaria que refleja el alejamiento de lo humano a su singularidad. Por esto, una adecuada armonía entre el impulso formal y el impulso sensible, entre el hombre como abstracción y el hombre con sus sentires singulares, es una demanda de la época.

Se expuso desde Schiller la posibilidad de justificar en la formación estética: la capacidad de producir o apreciar cosas bellas, una vía de confluencia armónica entre forma y sensibilidad. Con la expresión forma viva que es el equilibrado acuerdo entre forma $y$ contenido, entre el impulso formal y el impulso sensible, en términos de Schiller: la belleza, se pudo advertir en la existencia de cualidades del mundo en las que de hecho se da el equilibro entre estos dos impulsos. Tomar las cualidades bellas de los objetos, hechos, sucesos o acciones del mundo no solo como un fin en sí mismo, sino como una manera de adiestrar al intelecto en la armonía entre lo abstracto y lo singular, entre el entendimiento y la sensibilidad, se sugiere como un promisorio camino que conduce al balance integral del intelecto humano.

\section{Referencias bibliográficas}

Borges, J., L. (2014). Obras completas, tomo III. Emecé: Bogotá.

Descartes, R. (2010). Las meditaciones metafísicas. Bogotá: Universidad Nacional de Colombia.

Hume, D. (2008). Tratado de la naturaleza humana. Madrid: Tecnos.

Kant, I. (1784). ¿Qué es la ilustración? Recuperado de:http://pioneros.puj.edu.co/lecturas/ interesados/QUE\%20ES\%20LA\%20 ILUSTRACION.pdf 
Kant, I. (2006). Crítica de la razón pura. México: Taurus.

Mann, T. (1983). La muerte en Venencia. Bogotá: Oveja Negra.

Safranski, R. (2011). Goethe y Schiller, historia de una amistad. Barcelona: TusQuets.

Schiller, F. (1795). Cartas sobre la educación estética del hombre. Recuperado de: https:// clasesparticularesenlima.files.wordpress. com/2015/10/schiller-cartas-sobre-la-educacic3b3n-estc3a9tica-del-hombre-1795.pdf 